\title{
Supporting dispatch decisions for the fire and rescue services
}

\author{
Tobias Andersson Granberg, Jonas Lundberg, Anna Ulander and Granlund Rego
}

\author{
Linköping University Post Print
}

\section{Tweet}

N.B.: When citing this work, cite the original article.

Original Publication:

Tobias Andersson Granberg, Jonas Lundberg, Anna Ulander and Granlund Rego, Supporting dispatch decisions for the fire and rescue services, 2015, 2015 IEEE 18th International Conference on Intelligent Transportation Systems, 2562-2567.

http://dx.doi.org/10.1109/ITSC.2015.412

Copyright: (C2015 IEEE. Personal use of this material is permitted. However, permission to reprint/republish this material for advertising or promotional purposes or for creating new collective works for resale or redistribution to servers or lists, or to reuse any copyrighted component of this work in other works must be obtained from the IEEE.

\section{http://ieeexplore.ieee.org/}

Postprint available at: Linköping University Electronic Press

http://urn.kb.se/resolve?urn=urn:nbn:se:liu:diva-128628 


\section{Supporting dispatch decisions for the fire and rescue services}

\author{
Tobias A. Granberg, Jonas Lundberg, Anna Ulander \\ Dept. Science and Technology \\ Linköping University \\ Norrköping, Sweden \\ tobias.andersson@liu.se
}

\author{
Rego Granlund \\ SICS Swedish ICT \\ Linköping, Sweden
}

\begin{abstract}
Decision support tools for efficient dispatching of fire and rescue resources are developed and evaluated. The tools can give suggestions about which resources to dispatch to new accidents, and help the decision makers in evaluating the current preparedness for handling future accidents. The tools are evaluated using simulation game based experiments, with players from the fire and rescue services. The results indicate that the tools can help the fire and rescue services in identifying the closest resources to new accidents, and to select resources that preserve the preparedness in the area. However, the results also indicate that there is a risk that the tools increase the decision time.
\end{abstract}

Keywords-Emergency management; Fire and rescue services; Dispatch; Decision support system

\section{INTRODUCTION}

The Swedish fire and rescue services (FRS) have traditionally used a static form of planning and resource management. Fire stations are located in populated areas, and the firefighters wait at the station until an alarm is received. This makes it easy to design dispatch strategies; each station gets a pre-computed area of responsibility. For an event occurring in this area, the corresponding station is alerted. The dispatch is usually done with predetermined response units, meaning that a fixed crew has already been assigned to a vehicle, e.g. five firefighters that man a fire engine or two firefighters manning a ladder vehicle.

Recently, a new way of planning has started to gain ground in Sweden, where the firefighters are divided into smaller groups. These smaller units can be used for preventive work, or for maintaining a high level of preparedness by ensuring that they are strategically located close to where new accidents may occur. The closest available resource is usually dispatched to a new accident, and it may be necessary to dispatch more than one unit to obtain the resources necessary for a full response. Furthermore, it has become more common to use new types of resources as first response units. These can range from being personnel at the FRS currently doing other work than emergency response (e.g. education), to home care nurses assisting the FRS in medical emergencies.

The research presented in this paper was partly funded by the Swedish Civil Contingencies Agency
This new planning and control strategy generates benefits such as shorter first response times, improved coverage and the possibility to do more preventive work. However, the complexity of the planning situation also increases. Instead of knowing that the resources are available at the station, the decision makers have to keep track of a large number of small units spread over the area. Thus, it becomes more complicated to make decisions about which resources to dispatch to new accidents and also to assess the preparedness in the area (i.e. evaluate the current ability to respond to new accidents).

The aim of the research presented in this paper, is to develop decision support tools that can facilitate the dispatch of fire and rescue service resources. The usefulness of the tools is tested and evaluated using simulation game based experiments.

To determine pre-computed areas of responsibility, response time analyses of various complexity can be made. First however, locations for the stations have to be determined, a problem that has been extensively studied (see e.g. [1]). In most of these models, it is assumed that the closest station or resource will provide the response - and this is also the most common dispatch rule in practice even though it has been shown, e.g. in [2], that it sometimes is better to dispatch units further away from the event. This is utilized in Sweden, where a priority based system is used for emergency medical services (EMS). It is common that less urgent calls can be assigned to ambulances with a longer travel time than the shortest to preserve the preparedness. In [3], the authors develop a preparedness measure that is used as a base for EMS dispatching and relocation models. While the work we present here share similarities with [3], the situation is more complex for FRS than for EMS. This is because ambulances often can be assumed to be homogenous and interchangeable, while FRS resources cannot. The same applies for the demand, where an urgent EMS call often can be assumed to be handled by any (although sometimes multiple) ambulance. For FRS, different accidents may require completely different resources. The dispatching strategies in [3], has been further developed e.g. in [4].

Furthermore, Swersey [5] developed a decision rule for how many vehicles to send to a certain incident, considering the urgency of the incident, the expected alarm rate in the surrounding area and the number of available vehicles in the 
area. An extension to this model is presented in [6]. While computer generated decision rules are essential for an efficient dispatch, they also have to be incorporated into a decision support system to be of practical use for the decision makers. This is considered e.g. in [7] where both assignment and routing of the vehicles are supported, and in [8] where an integrated emergency response fleet deployment system is presented. The system allows emergency vehicles to switch routes and be reassigned to new emergency calls that are more urgent than the current, as well as to change routes based on real-time traffic information. However, to the best of our knowledge, no dispatch support system for fire and rescue services capable of handling multiple types of vehicles, personal competences and different accident types have been previously presented in the literature.

\section{A DECISION SUPPORT SYSTEM FOR FIRE AND RESCUE SERVICES}

The dispatch support tools presented here are meant to facilitate efficient planning and control of fire and rescue resources, where the resources are different types of vehicles and firefighters with different sets of competences. They are integrated into a decision support system (DSS), where the core of the system is a geographical information system (GIS) called C3Resuce (based on C3Fire [9]), which gives the user an overview of the area and of all the resources. The user controls and manages the resources through the user interface (UI) SMOKE. Via SMOKE, the user can assign firefighters to vehicles and dispatch the resources to accidents and other missions.

Both in the map (C3Rescue) and in SMOKE (the UI), the user has an overview of all the resources, including locations, vehicle types and the firefighters' competences. A small extract from the map is shown in Fig. 1. The map illustrates all the resources and their current locations. The squares with a red frame are full-time stations, i.e. stations with full-time firefighters, and those with an orange frame are part-time fire stations. Full-time firefighters typically have a call out time (the time from when an alarm is received until they are supposed to start travelling towards the accident site) of 90 seconds. Part time firefighters have other jobs, and therefore first have to go to the fire station to equip. Their call out time is usually 5-6 minutes.

The small ovals represent firefighters and are colored differently depending on their competences. For example, the oval with a red top is a team leader that possess the competences First response, Basic firefighting, BA (Breathing apparatus) team leading, and Team leading, i.e. four competences in all. The oval with a black top, is a basic firefighter, and can do First response and Basic firefighting. Other competences include Ladder vehicle operation, Incident command, and External command. In Sweden, it is common that you have between one and four competences.

The small numbered rectangles represent vehicles. The numbers represent both to which fire station a vehicle belongs as well as the vehicle type; e.g. vehicle 231 is a base vehicle (fire engine) belonging to station 23. 234 is a ladder vehicle and $23 \mathrm{~b}$ is a first response vehicle, both from station 23 . The user can easily check the type of the vehicle in SMOKE. When the resources are not present at the fire stations, there is a brief notation both in the map and in SMOKE regarding their current mission. The information in the map can be found in a square next to the resource locations. For example, 'Info B23' is a non-urgent information mission for two firefighters. Urgent missions have a red frame.

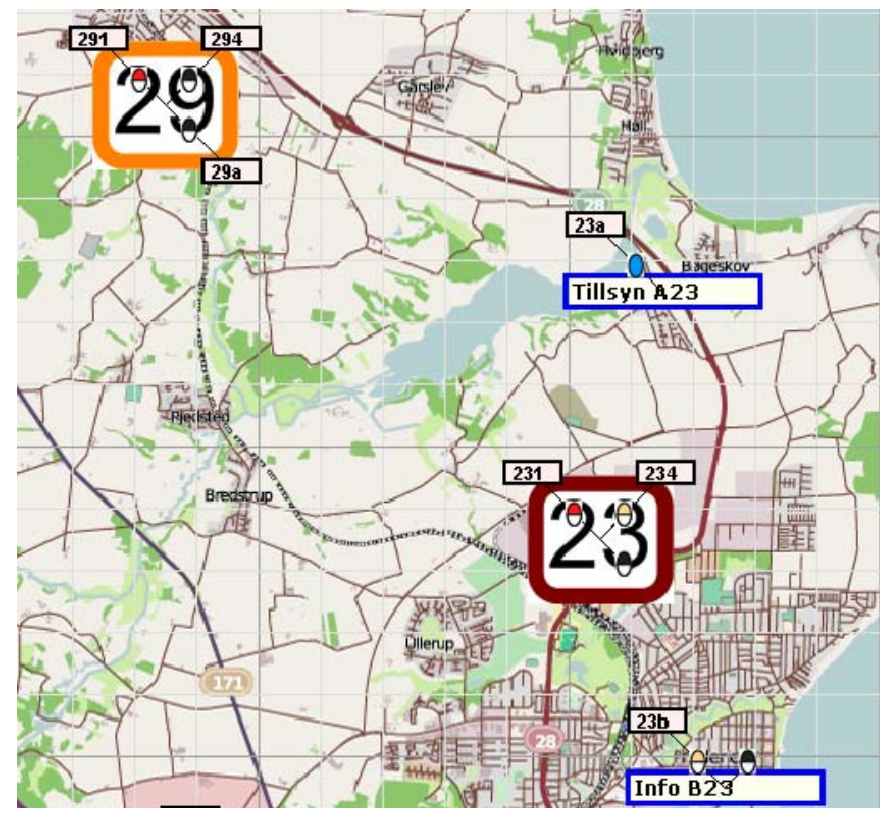

Fig. 1. An example of the digital map that illustrates the resources and their current locations

The firefighters and vehicles are handled individually in the system. This means that personnel is not pre-assigned to vehicles, which is common in practice. However, the reason for separating these, is to give the user the flexibility to construct non-traditional units, and enable a more dynamic way of planning. Thus, the user has to assign firefighters to vehicles before dispatching the vehicle to an event. To help the user select personnel and vehicles, a two specific tools have been incorporated into the DSS. The first tool can calculate and visualize the preparedness, while the second tool suggests suitable resources to send to different event types. The tools are further explained below.

\section{A. Calculation and visualization of preparedness}

Preparedness describes the ability to respond to accidents in the present and in the future. It is assumed to depend on the amount of available resources and their expected response times as well as the demand for service, which depends on the accident frequency. The preparedness for handling an accident of type $a$ in a zone $j$, is calculated as $p_{a j}=d_{a j}{ }^{*} t_{a j}$ where $d_{a j}$ is the demand for handling accidents of type $a$ in zone $j$ and $t_{a j}$ is the mean response time for the resources required to handle this accident type. As might be noted, the measure is a variant of the objective function for the $p$-median problem [10]. The main difference - and additional complexity - comes from the fact that we here take into account the response times for multiple resources, both vehicles and personnel. 
Since different types of accidents require different sets of resources, a set of required resources - an alarm plan - is defined for each accident type that is considered. As an example, vehicles required for a fire in a high rise building is defined as one fire engine and one ladder vehicle. Additionally, one team leader, one BA team leader, three firefighters and two ladder vehicle operators are needed - giving a total of seven personnel and two vehicle resources. $t_{a j}$ is calculated as the mean response time for the nine resources of the required type that has the shortest response time to zone $j$.

Furthermore, accidents also vary in frequency depending on the location. For each zone, a forecast, $f_{a j}$, of the expected number of accidents of type $a$ is produced. The demand for service is modelled as $d_{a j}=\left(f_{a j}\right)^{\gamma}$. This is because the resulting value $p_{a j}$ should correspond to the more abstract notion of preparedness, and must thus include a factor to balance the weight between frequency and response time. It was calibrated to correspond to preparedness judgments of FRS personnel, scaling the weight of the forecast down by $\gamma(<1)$.

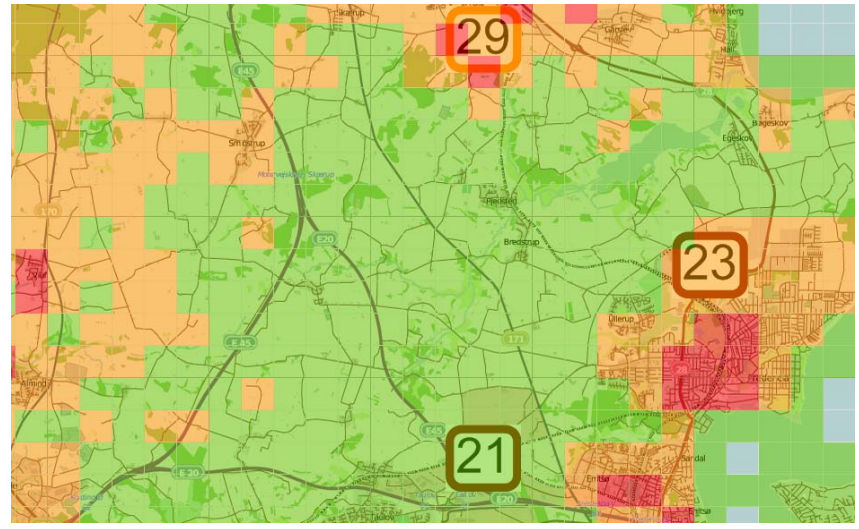

Fig. 2. Visualization of the preparedness

After the preparedness measure has been calibrated (the process for this, as well as more details on the development can be found in [11]) it can be visualized in the map (see Fig. 2). A zone with good preparedness is green, while a zone with inadequate preparedness is red. This directly gives the FRS information that can be used when dispatching resources. For instance, if possible, the user should rather dispatch resources currently located in green areas to new calls, than resources that currently are in, or close to, red areas. This, because the preparedness in an area typically deteriorates when resources that are close by becomes unavailable.

The preparedness $p_{a j}$ is calculated for each zone and each accident type. To get an indicator of the preparedness in the whole area, for all accident types, the most critical accident type in each zone is identified as the one for which the preparedness value is highest, i.e. $p_{j}=\max _{a}\left\{p_{a j}\right\}$. The area preparedness (PA) is then calculated as the sum over the $10 \%$ zones with the highest (worst) $p_{j}$ values. The motivation for this is that it should not be possible to obtain an adequate PA by only improving the situation for zones that already have an adequate preparedness (which is possible if the PA is chosen as the sum over all zones in the area). Likewise, only looking at the single zone with the highest value, like in [3], and ignoring all other zones, does not capture how the firefighters evaluate the preparedness in the area. It should be noted that the PA is only used in the analysis phase of this project, and not accessible directly in the DSS.

\section{B. Support for dispatch decisions}

The second tool can facilitate decisions about which resources to dispatch to incidents. When the preparedness is calculated for a particular type of accident in a specific zone, the resources that contribute to the preparedness are identified. These resources can also be illustrated by a mouse click on the map. The user can thus select any position on the map and get the resource information for a selected accident type. An example for a building fire accident is shown in Fig. 3.

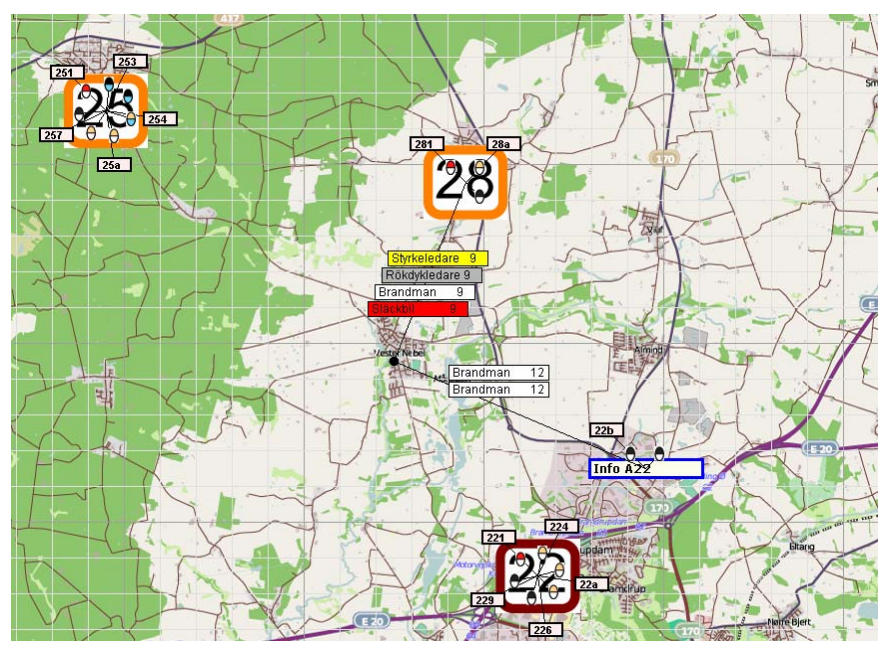

Fig. 3. The resources that contribute to the preparedness for building fires

For the selected position in Fig. 3 (the little black dot), the closest resources required for the accident type are located at fire station 28 and at Info A22. The response time is also presented for each specific resource. The response time for the resources located at station 28 is nine minutes, and the response time for the resources located at Info A22 is 12 minutes.

By defining new accident types with different resource requirements, it would be possible to vary the suggestions that are obtained from the system. For instance, it is possible to define the accident type "traffic accident with dangerous goods", and require a chem unit in addition to the standard response. While it is typically easy to keep track of special vehicles, since they are few and usually parked at the station, firefighters with special competences (e.g. full suit rescue) might be more difficult to keep track of, since they can be used for other tasks as well. The DSS helps the decision maker with this by visualizing the competences, and by showing where the closest resources can be found for that particular accident type.

Note that only the specified resources required for a full response for the selected accident type are shown when using the dispatch tool. It is possible that there are other resource types close to the selected position that are not listed in the initial requirements, but that may still be useful at the accident site. To make these resources visible to the user, it is possible 
to define the accident type "first response", which requires one personnel resource with the First response competence.

Another dispatch aid is available via SMOKE. The user can specify which and how many resources of each type that are desired for an event at a certain position. Then the tool gives the user a suggestion for which resources that should be dispatched in order to minimize the total response time.

\section{EXPERIMENTAL METHOD}

Two sets of experiments were performed to examine the differences between working without the decision support tools (Set 1), and having access to them (Set 2). In an experiment, decision makers from the FRS play a simulation game, in which they use the DSS. They are responsible for managing fire and rescue resources in a specific geographic region, and must assign resources for preventive work, dispatch resources to accidents, and maintain an adequate preparedness.

The region was based on an area in Denmark, which had been somewhat altered to better fit the purpose of the tests (e.g. some population were removed from border areas to avoid border issues and some vegetation were changed). It included two medium sized cities and around ten smaller communities, which corresponds to a medium sized real FRS organization in Sweden. The geographical area was divided into 2584 zones. Travel times between the zones was based on Open Street Map data (http://www.openstreetmap.org/) and were generated using Network Analyst in ArcMap 10. The forecast of expected number of accidents per year in each zone for each accident type was based on values for corresponding similar areas, and was validated as reasonable by experts from the FRS. To calculate the demand for service from the accident frequency, a value of $\gamma=0.25$ was determined together with experts from the FRS.

Three types of events were defined for preparedness calculations; traffic accidents, building fires and fires in high rise buildings. Furthermore, the "first response" type was also available, which means that the users could get preparedness visualization and dispatch suggestions for the three accidents types and also for a first response to the accidents. A total of 43 vehicles of seven different types were included in the experiments. Furthermore, the setting included 61 firefighters, which could have one or more of seven personnel competencies. Most firefighters had more than one competency, and they could have up to four.

To test the use of new types of resources, so called "day workers" were included. This is personnel employed by the FRS for nonemergency work, e.g. inspections or education. In the experiments they could be utilized for performing first response and give verbal information from the accident site, but not as a substitute for a firefighter (as they do not have the Basic firefighting competence).

In total seven experiments in each set were performed at 11 different FRS organizations. The same design and setup was used in each experiment, so that the previously mentioned difference between Set 1 and 2 would be measurable. Each experiment consisted of two scenarios, where in each scenario, the players (i.e. the decision makers) had to start by assigning resources to planned missions (education, inspections, etc). After this was done, the simulation game began, accidents occurred and the players had to dispatch resources to the accidents. The players had multiple goals in the experiment: a) to get the required resources to each accident as quickly as possible, b) to break as few planned missions as possible, and c) to preserve the preparedness as good as possible. Therefore it is not necessary that dispatching the closest units always was the best decision. Before the start of the experiment, the players were informed of these three goals. However, no instructions regarding prioritization were given, which means that the players used their own experience to make the necessary tradeoffs. Typically though, for most confirmed accidents in reality, the response time would be prioritized. In the two scenarios, a total of four accidents occurred. One experiment took around eight hours to perform.

An experiment requires three players; 1 . who makes the decisions; 2. who aids the decision maker and discusses the strategies and decisions. The main reason for this player is to enable verbal communication that can be captured on film; and 3 . who is in another room and plays all the roles of external personnel, e.g. firefighters reaching the accidents. A player was not allowed to participate in more than one experiment, in order to prevent learning effects. The players were FRS commanders selected by the FRS organizations. The experience of similar command and control (dispatching and preserving preparedness) that were performed in the experiments varied significantly, between zero and more than 30 years. Some of that variation was however reduced by the design with two players discussing the decisions before executing them.

In the first set of experiments, the players did not have access to the decision support tools, i.e. the preparedness visualizations and the dispatch suggestions. In the second set, they had access. In both sets, the players got support from the digital map (C3Rescue) and paper maps - where they could see the accident forecasts and the travel times between the fire stations - and the UI (SMOKE). Comparing the two sets of experiments, it was possible to assess the impact the decision support tools had on the operations.

\section{RESULTS}

To investigate whether access to the support tools could contribute to a more efficient dispatching of resources to the four accidents in each experiment, we studied:

1. Closest unit: How often the closest available unit also was the first unit to reach the accident site. This shows if the support tools (primarily the dispatch suggestion tools described in Section II.B) can help the user find the closest unit.

2. Decision time: How long time it took for the decision makers to dispatch the first unit. This shows if the players can make quicker decisions with help of the dispatch support tools.

3. Preparedness preservation: We measured the preparedness in the area directly after the players had finished dispatching resources, but before they had 
started to relocate resources to improve the preparedness. This shows if the support tools (primarily the preparedness visualization tool described in Section II.A) can help the user find "preparedness-robust" resources to dispatch, i.e. resources that, when they become unavailable, will not cause the preparedness to deteriorate significantly.

1 and 2 correspond to Goal a) in Section III, i.e. facilitating a short response time. For this, it is necessary to both select resources that can get to the accident quickly, and to make a quick decision. 3 correspond to Goal c). The fulfillment of Goal b) was also measured as how often units that were busy with nonemergency work, had to leave their current task. The results showed that players in both sets utilized these units, especially for the more resource consuming accidents. No significant difference between the experiment sets for this measure could be discerned.

In TABLE I. the proportion of times that the closest available unit was the first to reach the accident site is presented for each set of experiments. For Accident 1 (Building fire), the closest unit was sent in 3 out of $7(43 \%)$ experiments in Set 1, and 5 out of $7(71 \%)$ experiments in Set 2. For Accident 4, the players in Set 2 also dispatched the closest available unit more often than in Set 1, while for Accident 2 and 3, the proportion is equal for the two sets.

TABLE I. HOW OFTEN THE CLOSEST AVAILABLE UNIT ALSO WAS THE FIRST UNIT TO REACH THE ACCIDENT SITE

\begin{tabular}{ccccc}
\hline & \multicolumn{4}{c}{ Accidents } \\
Set & $\begin{array}{c}\text { 1. Building } \\
\text { fire }\end{array}$ & $\begin{array}{c}\text { 2. Drowning } \\
\text { accident }\end{array}$ & $\begin{array}{c}\text { 3. Traffic } \\
\text { accident }\end{array}$ & $\begin{array}{c}\text { 4. Apartment } \\
\text { fire }\end{array}$ \\
\hline 1 & $43 \%$ & $100 \%$ & $71 \%$ & $67 \%$ \\
2 & $71 \%$ & $100 \%$ & $71 \%$ & $100 \%$
\end{tabular}

This result indicates that the dispatch support tools helped the players find the closest unit to dispatch to the accidents. This was probably partly due to tools' ability to make the resources more visible to the player, but also to the direct access to travel times to the accident for the required resources. Without the tools, the players in Set 1 had to rely on the limited set of pre-computed travel times between the fire stations, together with their own estimations.

The time it took for the players to decide which unit to dispatch as the first unit is presented in TABLE II. While most of the time it took between one and two minutes, some players were very quick (like in Experiment 5), and others thought things over for a long time (like the players in Experiment 12).

Looking at the mean decision times, they are quite similar between the two sets for Accident 2 and 4. For Accident 1, the mean is lower in Set 2. One explanation for this might be that the players in the beginning of the experiment is unfamiliar with the system and need a longer time to achieve situational awareness. Possibly, the dispatch tools help the players in Set 2 to overcome this unfamiliarity.

Looking at Accident 3, the difference in mean decisions times between the sets are mainly due to the exceptionally large value in Experiment 12 (nothing exceptional happened to cause the long time though; the players just thoroughly discussed the entire response, before starting to dispatch). Moreover, the quick decision time in Experiment 5 further adds to the difference. Removing the largest and smallest values (Min and Max) from each set gives a mean time of 1:57 for Set 1 and 2:21 for Set 2, i.e. the difference is reduced but the mean time is still longer for Set 2.

TABLE II. HOW LONG TIME [MIN:SEC] IT TOOK FOR THE DECISION MAKERS TO DISPATCH THE FIRST UNIT

\begin{tabular}{|c|c|c|c|c|}
\hline \multirow[b]{2}{*}{ Experiment } & \multicolumn{4}{|c|}{ Accidents } \\
\hline & $\begin{array}{c}\text { 1. Building } \\
\text { fire }\end{array}$ & $\begin{array}{l}\text { 2. Drowning } \\
\text { accident }\end{array}$ & $\begin{array}{c}\text { 3. Traffic } \\
\text { accident }\end{array}$ & $\begin{array}{l}\text { 4. Apartment } \\
\text { fire }\end{array}$ \\
\hline 1 & $04: 10$ & $01: 48$ & $02: 40$ & $\mathrm{~N} / \mathrm{A}^{\mathrm{a}}$ \\
\hline 2 & $04: 27$ & 01:03 & $01: 55$ & $01: 54$ \\
\hline 3 & 03:04 & $01: 33$ & 02:03 & 01:03 \\
\hline 4 & $\mathrm{~N} / \mathrm{A}^{\mathrm{b}}$ & 01:04 & 03:16 & $01: 10$ \\
\hline 5 & $01: 42$ & $00: 38$ & $00: 40$ & $00: 54$ \\
\hline 6 & 03:06 & $01: 47$ & 02:04 & 01:06 \\
\hline 7 & $03: 32$ & $01: 42$ & 01:04 & $\mathrm{N} / \mathrm{A}^{\mathrm{b}}$ \\
\hline Mean & $03: 20$ & $01: 22$ & 01:57 & 01:13 \\
\hline Min & 01:42 & $00: 38$ & $00: 40$ & $00: 54$ \\
\hline Max & $04: 27$ & 01:48 & $03: 16$ & 01:54 \\
\hline 8 & $01: 20$ & $01: 11$ & $02: 56$ & $03: 26$ \\
\hline 9 & 01:43 & $01: 22$ & $02: 12$ & 01:06 \\
\hline 10 & $01: 43$ & $02: 38$ & $02: 57$ & 01:07 \\
\hline 11 & 01:41 & $01: 42$ & $01: 43$ & 01:10 \\
\hline 12 & $05: 40$ & $01: 17$ & 09:29 & $00: 20$ \\
\hline 13 & $02: 16$ & 02:07 & $01: 55$ & 01:30 \\
\hline 14 & 01:12 & $00: 43$ & $01: 36$ & $00: 51$ \\
\hline Mean & $02: 14$ & $01: 34$ & $03: 15$ & $01: 21$ \\
\hline Min & 01:12 & $00: 43$ & $01: 36$ & $00: 20$ \\
\hline Max & $05: 40$ & $02: 38$ & 09:29 & 03:26 \\
\hline
\end{tabular}

All in all, it is not possible to say with certainty whether or not the dispatch support tools facilitates quicker dispatch decisions. However, in three out of four accidents, the players in Set 1 are on average quicker to dispatch than the players in Set 2. This indicates that the support tools prolong the dispatch process, which might be explained by the fact that the players had to use them - and thus perform one more action - before dispatch was done.

The area preparedness (PA; as described in Section II.A) was measured to get an indication of how well the players were able to preserve the preparedness during the dispatch process. This was done after the players had finished dispatching resources to an accident. Since a specific accident required the same set of resources in each experiment, the same set of 
resources were also unavailable after the dispatch was finished in each experiment. Thus, the locations of the remaining available resources determined how well the players were able to preserve the preparedness, without additional relocation of resources.

TABLE III. AREA PREPAREDNESS MEASUREMENTS

\begin{tabular}{ccccc}
\hline & \multicolumn{4}{c}{ Accidents } \\
Set 1 & $\begin{array}{c}\text { 1. Building } \\
\text { fire }\end{array}$ & $\begin{array}{c}\text { 2. Drowning } \\
\text { accident }\end{array}$ & $\begin{array}{c}\text { 3. Traffic } \\
\text { accident }\end{array}$ & $\begin{array}{c}\text { 4. Apartment } \\
\text { fire }\end{array}$ \\
\hline Mean & 5026 & 5322 & 6047 & $6164^{\mathrm{c}}$ \\
Min & 4720 & 4949 & 5043 & 5588 \\
Max & 5507 & 5541 & 8935 & 4954552 \\
\hline Set 2 & & & & \\
\hline Mean & 5107 & 5332 & 5028 & 5596 \\
Min & 4780 & 4835 & 4633 & 5189 \\
Max & 5355 & 5554 & 5666 & 6060 \\
\hline $\begin{array}{c}\text { Difference } \\
\text { Set 2 - Set 1 } \\
\text { mean [\%] }\end{array}$ & $1.6 \%$ & $0.2 \%$ & $-16.9 \%$ & $-9.2 \%$ \\
\hline
\end{tabular}

In TABLE III. the mean, max and min PA is presented for each experiment set and accident. A lower value corresponds to better preparedness. Looking at the mean values, they are slightly better in Set 1 for Accident 1 and 2, while they are better in Set 2 for Accident 3 and 4. The extremely large max value in Set 1, Accident 4, is caused by a lack of ladder vehicles in Experiment 4 - the players assigned all available resources of that type to accidents, and thus made them unavailable. Since ladder vehicles are required for fires in high rise buildings, the preparedness for that accident type became severely affected, resulting in a very large preparedness value. The mean value for Set 1 is calculated without using this large value, but still the mean for Set 2 is $9.2 \%$ lower.

The large difference in preparedness values in the latter accidents might be due to the fact that many resources were required to handle the simultaneously occurring accidents 2,3 and 4 . Thus, available resources were becoming scarce at the end of the scenario. It is possible that the decision support tools were able to help the players carefully plan how to best use this limited set of resources. Overall, the results indicate that support tools can help the users select resources for dispatch in a way that preserves the preparedness.

Another possible indicator of the usefulness of the dispatch support tools is of course the response times to the accidents, i.e. if using the tools in the long term will lower the mean response time. We also measured the response times for the accidents, but as expected, the number of experiments needed to draw statistically sound conclusions is much higher than what was possible to perform during this project. Still, it is an interesting possibility for future research.

\section{CONCLUSIONS}

In this paper we describe the development and evaluation of decision support tools capable of facilitating the dispatching of fire and rescue resources. Based on an experimental study of tool usage in realistic scenarios by experienced rescue services personnel, our results show that the tools can help the users in selecting the closest resources to send to an accident, and also help them select resources so that the preparedness for handling new accidents is preserved. However, the results also indicate that the time it takes to decide which resource to dispatch might increase when the tools are introduced.

The increased decision time might be due to the simple fact that using the tools takes some time, especially when they are new to the users. If introducing these kinds of tools into the daily work, that problem might possibly disappear, since the users would get more proficient in using them. This could be tested by running a third set of experiments, where the players get thorough training and become proficient in using the system. Then it would be possible to see whether familiarity with the system is a critical factor. Another possibility is of course to construct more user friendly and computationally efficient tools, that more quickly can give better support, and that are easier to understand and implement. Comments from the fire and rescue service personnel participating in the experiments indicated that these kinds of support tools are much wanted, and that more research providing a base for how they should be constructed, tested and used is needed.

\section{REFERENCES}

[1] A.J. Swersey, "The Deployment of Police, Fire, and Emergency Medical Units," in Handbooks in OR \& MS, vol. 6, S. M. Pollock, M. H. Rothkopf and A. Barnett, Eds. Elsevier Science B.V., 1994, pp. 151200.

[2] M. C. Carter, J. M. Chaiken, and E. Ignall, "Response Areas for Two Emergency Units," Ops. Res., 1972, vol. 20(3), pp. 571-594.

[3] T. Andersson and P. Värbrand, "Decision support tools for ambulance dispatch and relocation", J Oper Res Soc, 2007, vol 58(2), pp. 195-201.

[4] S. Lee, "The role of preparedness in ambulance dispatching", J Oper Res Soc, 2011, vol. 62, pp. 1888-1897.

[5] A. J. Swersey, "A Markovian Decision Model for Deciding How Many Fire Companies to Dispatch," Man. Sci., 1982, vol. 28(4), pp. 352-365.

[6] E. Ignall, G. Carter, and K. Rider, (1982) "An Algorithm for the Initial Dispatch of Fire Companies,” Man. Sci., 1982, vol. 28(4), pp. 366-378.

[7] A. Weintraub, J. Aboud, C. Fernandez, G. Laporte and E. Ramirez, "An emergency vehicle dispatching system for an electric utility in Chile", J Oper Res Soc, 1999, vol. 50, pp. 690-696

[8] A. Haghani and S. Yang, "Real-Time Emergency Response Fleet Deployment: Concepts, Systems, Simulation \& Case Studies", in Dynamic Fleet Management, V. Zeimpekis, C. D. Tarantilis, G. M. Giaglis and I. Minis, Eds. New York Springer, 2007, pp. 133-162.

[9] R. Granlund, "Monitoring experiences from command and control research with the C3Fire microworld," Cogn. Technol. Work. 2003, vol. 5(3), pp. 183-190.

[10] S. L. Hakimi, "Optimum distribution of switching centers in a communication network and some related graph theoretic problems," Oper. Res. 1965, vol. 13, 462-475.

[11] A. Ulander, "Optimization based decision support tools for fire and rescue resource planning", Linköping Studies in Science and Technology, Thesis No 1706, Licentiate Thesis, 2015. 